\title{
Gyrofluid computation of magnetic perturbation effects on turbulence and edge localized bursts
}

\author{
J. Peer ${ }^{1}$, A. Kendl ${ }^{1}$, T.T. Ribeiro ${ }^{2}$ and B.D. Scott ${ }^{2}$ \\ ${ }^{1}$ Institut für Ionenphysik und Angewandte Physik, Universität Innsbruck, Austria \\ ${ }^{2}$ Max-Planck-Institut für Plasmaphysik, Garching, Germany
}

\begin{abstract}
The effects of non-axisymmetric resonant magnetic perturbation fields (RMPs) on saturated drift-wave turbulence and on ballooning mode bursts in the edge pedestal of tokamak plasmas are investigated by numerical simulations with a nonlinear six-moment electromagnetic gyrofluid model including zonal profile evolution. The vacuum RMP fields are screened by plasma response currents, so that magnetic transport by perturbed parallel motion is not significantly changed. Radial transport of both particles and heat is dominated by turbulent convection even for large RMP amplitudes, where formation of stationary convective structures leads to edge profile degradation. Modelling of ideal ballooning mode unstable edge profiles for single bursts including RMP fields causes resonant mode locking and destabilization.
\end{abstract}




\section{INTRODUCTION}

In future tokamaks like ITER, the heat flux associated with edge-localised modes (ELMs) is estimated to seriously damage the plasma facing components (PFCs) [1 3]. Thus, methods for the suppression or at least effective mitigation of ELMs are essential for economically viable steady state operation of tokamaks. One of the most promising techniques to control ELMs is the external application of non-axisymmetric resonant and non-resonant magnetic perturbations which were found to increase the ELM frequency and to reduce the heat load on the PFCs [4 11]. Models for the physics underlying the ELM mitigation by resonant magnetic perturbation fields (RMPs) assume an RMP-induced formation of ergodic magnetic field regions which modify the radial transport and hence decrease the edge pressure gradient below the peeling-ballooning instability threshold [12 14].

Numerical computations on the effects of RMPs face the problem that present firstprinciples-based edge turbulence models are not able to obtain a self-consistent H-mode edge transport barrier with realistically steep flow and pressure profiles. Thus, a self-consistent treatment of the interaction between ELMs and RMPs is not possible. In contrast, present simulation codes allow for well-founded investigations on the effects of RMPs in L-mode edge turbulence conditions. Previous computational studies on the effects of RMPs on turbulence where based on two- and four-field reduced MHD models [15, 16] and four-field drift-fluid models [17-19]. The gyrofluid approach presented in this work allows to include self-consistent electron and ion temperature dynamics. Thus, a more realistic treatment of the radial heat transport becomes possible.

Bearing in mind the consistency limitations concerning H-mode states, we then further examine two models for the edge transport barrier. As one approach, RMP fields are included in the standard (unmitigated) "H-mode"-like profile model described in Refs. [21, 22] for gyrofluid computation of edge localized ideal ballooning mode bursts. It is found that this standard profile scenario does not allow to reproduce direct mitigation of ideal ballooning ELMs by RMPs.

The work is organised as follows. After the introduction in sec. 1, the gyrofluid computational model, the numerical setup and the method used to implement RMPs are presented in sec. 2. The turbulence computation results are evaluated and analysed in sec. 3, and the ELM simulation methods and results are discussed in sec. 4. Conlusions are given in sec. 5 . 


\section{COMPUTATIONAL GYROFLUID AND GEOMETRY MODEL INCLUDING RMPS}

The computations presented are carried out with the nonlinear gyrofluid electromagnetic model and code GEMR [23]. The model includes six moment equations each for electrons and ions (labelled with $z \in\{\mathrm{e}, \mathrm{i}\}$ ) which are coupled by a polarisation equation and an induction equation. The dependent variables are density $n_{z}$, parallel velocity $u_{z \|}$, parallel temperature $T_{z \|}$, perpendicular temperature $T_{z \perp}$, parallel component of the parallel heat flux $q_{z\|\| \|}$, perpendicular component of the parallel heat flux $q_{z \| \perp}$, electric potential $\phi$ and parallel magnetic potential $A_{\|}$. The full set of model equations is presented in Refs. [21, 23].

The model uses normalised quantities, where the perpendicular spatial scales are given in units of the minor plasma radius $a$. The time scale is normalised by $a / c_{\mathrm{s} 0}$, where $c_{\mathrm{s} 0}=\sqrt{T_{\mathrm{e} 0} / M_{\mathrm{i}}}$ is the reference plasma sound speed. Here, $M_{\mathrm{i}}$ denotes the ion mass and $T_{\mathrm{e} 0}$ is the reference electron temperature. The dependent variables are normalised by $n_{z} \leftarrow n_{z} / n_{z 0}$, $T_{z} \leftarrow T_{z} / T_{z 0}, u_{z \|} \leftarrow u_{z \|} / c_{\mathrm{s} 0}, q_{z \|} \leftarrow q_{z \|} /\left(n_{z 0} T_{z 0} c_{\mathrm{s} 0}\right), \phi \leftarrow(e \phi) / T_{\mathrm{e} 0}, A_{\|} \leftarrow A_{\|} /\left(\rho_{\mathrm{s} 0} \beta_{\mathrm{e} 0} B_{0}\right)$, where $n_{z 0}$ represents the reference density, $T_{z 0}$ is the reference temperature, $e$ denotes the elementary charge, $B_{0}$ represents the equilibrium magnetic flux density, $\rho_{\mathrm{s} 0}=c \sqrt{M_{\mathrm{i}} T_{\mathrm{e} 0}} /\left(e B_{0}\right)$ is the drift scale and $\beta_{\mathrm{e} 0}=4 \pi p_{\mathrm{e} 0} / B_{0}^{2}$ is the reference value for the electron dynamical beta. Here, $p_{\mathrm{e} 0}=n_{\mathrm{e} 0} T_{\mathrm{e} 0}$ denotes the reference electron pressure. The magnetic flux density is normalised by $B_{0}$.

The main model parameters are the electron dynamical beta $\beta_{\mathrm{e} 0}$, the normalised drift scale $\delta_{0}=\rho_{\mathrm{s} 0} / a$ and the collisionality $\nu_{\mathrm{e} 0}=a / c_{\mathrm{s} 0} \tau_{\mathrm{e} 0}$, where $\tau_{\mathrm{e} 0}$ denotes the reference value for the Braginskii electron collision time [21, 23]. The model dynamically evolves the fluctuating and the zonal and axisymmetric sideband components of the dependent variables. Here, zonal denotes the flux-surface average and sideband denotes the axisymmetric but non-zonal component. The inner (source) radial boundaries for the axisymmetric part of the variables are given by zero Neumann conditions. The inner radial boundaries for the fluctuating part of the variables as well as the outer (sink) radial boundaries are given by zero Dirichlet conditions. The computational domain includes an edge pedestal closed-fluxsurface (CFS) region with consistent quasi-periodic parallel-perpendicular boundary conditions and a scrape-off-layer (SOL) region where the parallel boundary conditions represent a Debye sheath limiter placed at the bottom side of a circular torus [24, 25]. 
The evolution of the profiles is self-consistently coupled to the magnetic Shafranov equilibrium for circular flux surfaces. Both the safety factor $q$ and the Shafranov shift are evolved in each time step [26].

The geometry is described in terms of field-aligned, unit-Jacobian Hamada coordinates $\left(x, y_{k}, s\right)$ through

$$
\begin{aligned}
x & =V=2 \pi^{2} R_{0} r^{2}, \\
y_{k} & =y-\alpha_{k}=q \theta-\zeta-\alpha_{k}, \\
s & =\theta
\end{aligned}
$$

where $V$ is the volume enclosed by the flux surface with major radius $R_{0}$ and minor radius $r$, and $\theta(0 \leq \theta<1)$ and $\zeta(0 \leq \zeta<1)$ are the unit-cycle poloidal and toroidal Hamada angles (see Ref. [21] for their definition). $V$ is given in units of $a^{3}$, and $R_{0}$ and $r$ are normalised by $a$. In order to avoid magnetic shear deformation of grid cells, the $y$-coordinate is shifted by $\alpha_{k}=q \theta_{k}+\Delta \alpha_{k}$, i.e. $\Delta \alpha_{k}$ is chosen to make $\boldsymbol{\nabla} x$ and $\boldsymbol{\nabla} y_{k}$ locally orthogonal at $\theta=\theta_{k}$ [27].

The initial magnetic equilibrium is computed from a prescribed safety factor profile $q_{0}(x)$. The temporal evolution of the Shafranov shift and $q(x)$ are determined by the PfirschSchlüter current and the associated axisymmetric component of $A_{\|}[26]$. The Shafranov shift is incorporated into the coordinate grid by modifying the metric elements according to the $s$ - $\alpha$ model. The resulting relevant part of the coordinate metric is given by

$$
\begin{aligned}
& g^{x x}=\boldsymbol{\nabla} x \cdot \boldsymbol{\nabla} x=(2 \pi)^{4}\left(R_{0} r\right)^{2}+\mathcal{O}(\varepsilon) \\
& g_{k}^{y y}=\boldsymbol{\nabla} y_{k} \cdot \boldsymbol{\nabla} y_{k}=\frac{q^{2}}{(2 \pi r)^{2}}+\mathcal{O}(\varepsilon) \\
& g_{k}^{x y}=\boldsymbol{\nabla} x \cdot \boldsymbol{\nabla} y_{k}=0 \quad \text { at } \theta=\theta_{k}
\end{aligned}
$$

where $g^{x x}$ and $g_{k}^{y y}$ are given to lowest order in $\varepsilon=r / R_{0}$.

RMPs are included by adding a time-independent perturbation to the intrinsic parallel magnetic potential (i.e. $A_{\|} \rightarrow A_{\|}+A_{\mathrm{p}}$ ). The perturbation potential is defined as [17]

$$
\begin{aligned}
A_{\mathrm{p}} & =-A_{0} \sum_{m}(-1)^{m} g(r) \cos [2 \pi(m \theta-n \zeta)] \\
& =-A_{0} \sum_{m}(-1)^{m} g(r(x)) \cos \left\{2 \pi\left[m s-n\left(q s-y_{k}-\alpha_{k}\right)\right]\right\}
\end{aligned}
$$

where $m$ and $n$ are the poloidal and toroidal mode number of the perturbation, $A_{0}$ is an amplitude factor and $g(r)$ denotes the radial envelope of the perturbation potential. For 
a perturbation including several poloidal mode numbers, the factor $(-1)^{m}$ can be used to define the poloidal localization of the perturbation field. The generation of RMPs by external perturbation coils requires RMPs to satisfy the constraint of zero additional plasma current. Using Ampère's law, this yields to the Poisson equation

$$
-\nabla_{\perp}^{2} A_{\mathrm{p}}=-\frac{1}{J} \frac{\partial}{\partial x^{\mu}} J g_{\perp}^{\mu \nu} \frac{\partial}{\partial x^{\nu}} A_{\mathrm{p}}=J_{\mathrm{p}} \equiv 0
$$

where $J$ is the coordinate Jacobian and $g_{\perp}^{\mu \nu}$ denotes metric elements. The time-dependent, global geometry implies that Eq. (9) has to be solved numerically in each time step.

The initial state for the turbulence computations is based on typical ASDEX Upgrade (AUG) L-mode profiles. The AUG plasma values are here used only to introduce a typical medium-size tokamak reference scenario, and no direct comparison with experimental results is at present intended on grounds of several idealisations present in the model, like, for example, the use of simplified geometry and local (linearised) polarisation.

The mid-pedestal reference values for density, temperature and magnetic equilibrium field are $n_{\mathrm{e} 0}=n_{\mathrm{i} 0}=2 \times 10^{-19} \mathrm{~m}^{-3}, T_{\mathrm{e} 0}=T_{\mathrm{i} 0}=100 \mathrm{eV}$ and $B_{0}=2.5 \mathrm{~T}$. The resulting reference values for plasma beta, electron collisionality, drift scale and Lundquist number are $\beta_{\mathrm{e} 0}=6.4 \times 10^{-5}, \nu_{\mathrm{e} 0}=1.6 \times 10^{6} \mathrm{~s}^{-1}, \rho_{\mathrm{s} 0}=5.8 \times 10^{-4} \mathrm{~m}$ and $S_{0}=1.2 \times 10^{7}$.

We consider a circular toroidal tokamak with major radius $R_{0}=1.65 \mathrm{~m}$ and minor radius $a=0.5 \mathrm{~m}$. The simulation domain has a radial extension of $\Delta r=0.07 \mathrm{~m}$ around the separatrix at $r=a$. The initial safety factor profile is prescribed by $q_{0}(r)=1.42+3.31(r / a)^{2}$ which yields $q$-values within the interval $4.28 \leq q_{0} \leq 5.21$ and a magnetic shear in the range $1.34 \leq \hat{s}_{0} \leq 1.46$. The initial gradient lengths of density and temperature are $L_{n}=0.07 \mathrm{~m}$ and $L_{T}=L_{\perp}=L_{n} / 2$.

We use a space resolution of $x \times y_{k} \times s=64 \times 512 \times 16$ grid points, where $x$ denotes the radial, $y_{k}$ the perpendicular (Clebsch angle) and $s$ the poloidal direction. The resulting resolution includes perpendicular scales down to twice the drift scale. The time resolution is $0.002 a / c_{\mathrm{s}}=1.4 \times 10^{-8} \mathrm{~s}$. The initial pedestal profiles for density and temperature are prescribed by $n(r) / n_{0}=\left(L_{\perp} / L_{n}\right) g_{0}(r)$ and $T(r) / T_{0}=\left(L_{\perp} / L_{T}\right) g_{0}(r)$ with $g_{0}(r)$ modelled as $g_{0}(r)=0.5-0.5 \sin \{2 \pi[r-(a-\Delta r / 4)] / \Delta r\}$ for $a-\Delta r / 2 \leq r \leq a$. The erosion of the pedestal profiles by radial transport is counteracted by a time-independent source flux which models the radial inflow of particles and heat from the core region. Thus, the simulations saturate at a statistically stationary turbulent state. 


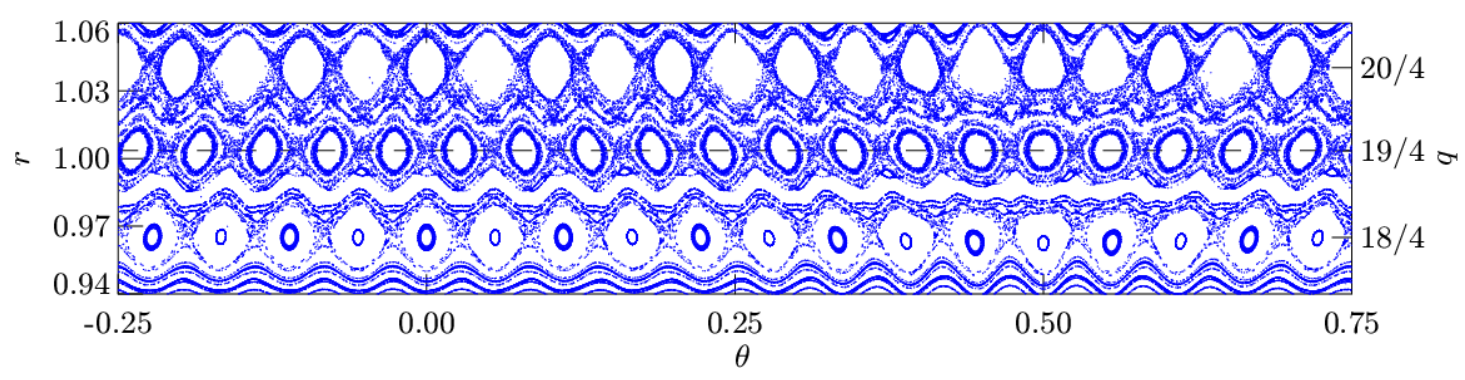

(a) $A_{0}=100$

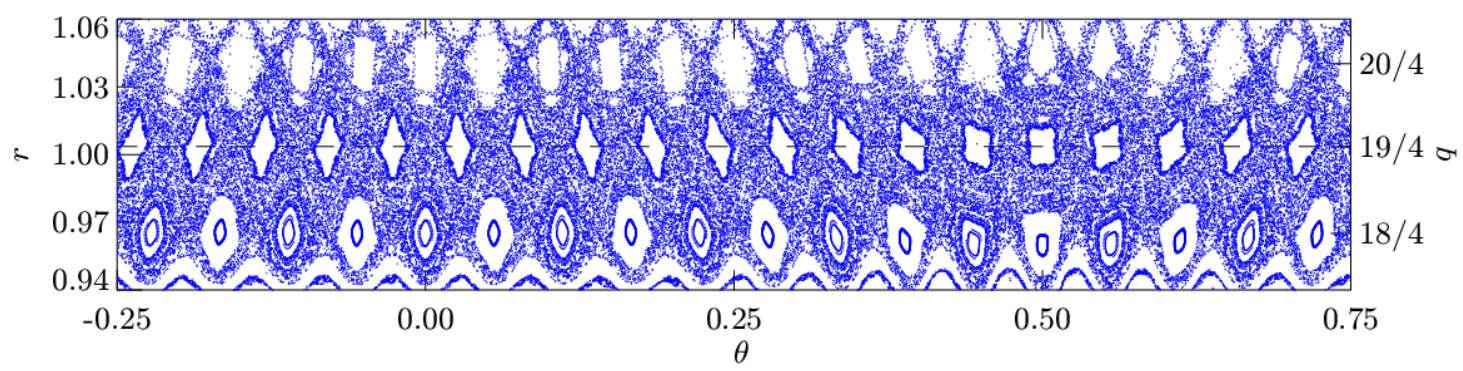

(b) $A_{0}=200$

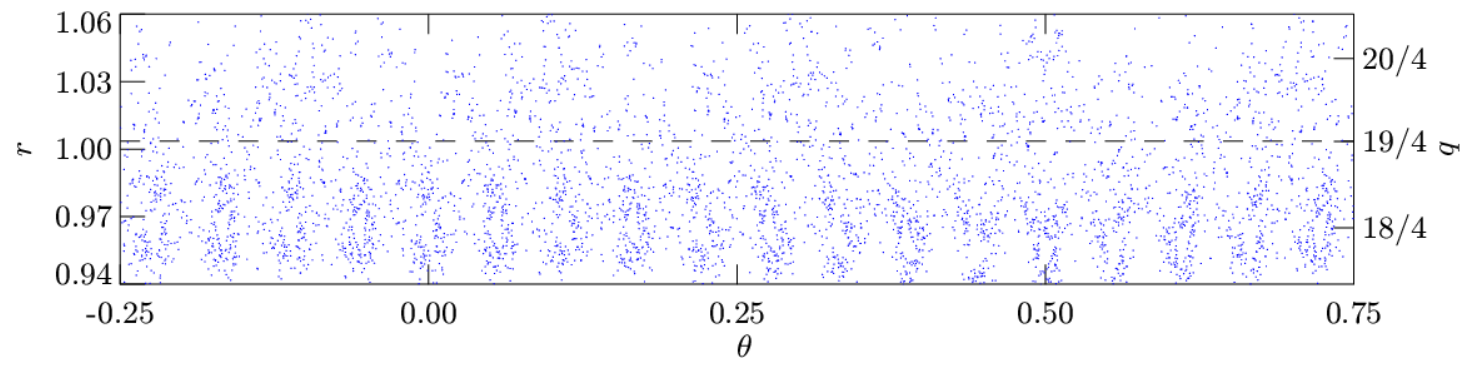

(c) $A_{0}=400$

FIG. 1: Poloidal Poincaré sections of the vacuum RMP fields for the perturbation amplitudes $\left(A_{0}=100,200,400\right)$ used in the simulations. 40 magnetic field lines were followed over 4000 toroidal turns. In (c), most of the field lines leave the simulation domain after some 100 toroidal turns. The dashed lines mark the $q=19 / 4$ position close to the separatrix (at $r=1$ ).

The computations include RMP fields with three different perturbation amplitudes $A_{0}$ and helicity components $m / n=18 / 4, m / n=19 / 4$ and $m / n=20 / 4$. The magnetic perturbation potential is prescribed to be equal on both radial boundaries, with $g(a-$ $\Delta r / 2)=g(a+\Delta r / 2)=1$. The applied perturbation amplitudes correspond to $9.3 \times 10^{-6} \mathrm{Tm}$ $\left(A_{0}=100\right), 1.9 \times 10^{-5} \mathrm{Tm}\left(A_{0}=200\right)$ and $3.7 \times 10^{-5} \mathrm{Tm}\left(A_{0}=400\right)$ and yield magnetic perturbation fields of order $10^{-3} \mathrm{~T}$. The RMP fields are located on the high-field side at 
$\theta=0.5$. Fig. 1 shows poloidal Poincaré sections of the vacuum RMP fields. For $A_{0}=100$ the RMP-induced islands at resonant flux surfaces are well separated. For $A_{0}=200$ islands overlap and ergodic regions between the islands are formed. A further increase of the perturbation amplitude to $A_{0}=400$ results in a strongly ergodised magnetic field.

\section{L-MODE TURBULENCE SIMULATIONS}

The simulations are evaluated in terms of space and time averages over statistically stationary turbulent states. Time averages are taken over an interval of $\Delta t=1000 a / c_{\mathrm{s}}=$ $7.2 \times 10^{-3}$ s. For the evaluation the dependent variables are separated as

$$
f\left(x, y_{k}, s, t\right)=\left\langle f\left(x, y_{k}, s, t\right)\right\rangle_{t}+\tilde{f}_{t}\left(x, y_{k}, s, t\right)
$$

where $\left\langle f\left(x, y_{k}, s, t\right)\right\rangle_{t}$ and $\tilde{f}_{t}\left(x, y_{k}, s, t\right)$ denote the stationary and the temporally fluctuating part of a dependent variable $f\left(x, y_{k}, s, t\right)$. Spatial fluctuations of a dependent variable are computed with respect to its toroidal mean as $\tilde{f}_{x}\left(x, y_{k}, s, t\right)=f\left(x, y_{k}, s, t\right)-\left\langle f\left(x, y_{k}, s, t\right)\right\rangle_{y_{k}}$.

\section{A. Screening of RMPs by plasma response currents}

The plasma response to externally applied RMPs consists of parallel currents which alter the vacuum structure of the perturbation fields. Fig. 2 shows the RMP-induced variation in the stationary component of the parallel plasma current fluctuations in the poloidal plane. It is found that RMPs give rise to current fluctuations at resonant flux surfaces. The fluctuation amplitudes increase quasi-linearly with the perturbation amplitude. Due to lower fluctuation amplitudes in the SOL, the effect is more pronounced in the CFS region.

The corresponding Poincaré plots, computed from the stationary component of the magnetic flutter field, are shown in Fig. 3. The magnetic island structure exhibits the mode number of the imposed RMP fields but the islands are poloidally shifted by about half a poloidal island. Moreover, the radial extension of the islands is reduced. Thus, the simulations show that the plasma response currents are very effective at screening the vacuum RMP fields. The magnetic islands associated with the vacuum RMP fields are closed and reopened with decreased amplitude at a poloidally shifted position. The screening effect can furthermore be quantified by the amplitude of the perpendicular magnetic flutter. For $A_{0}=400$ the magnetic flutter is reduced by $73 \%$ with respect to the vacuum RMP fields. 


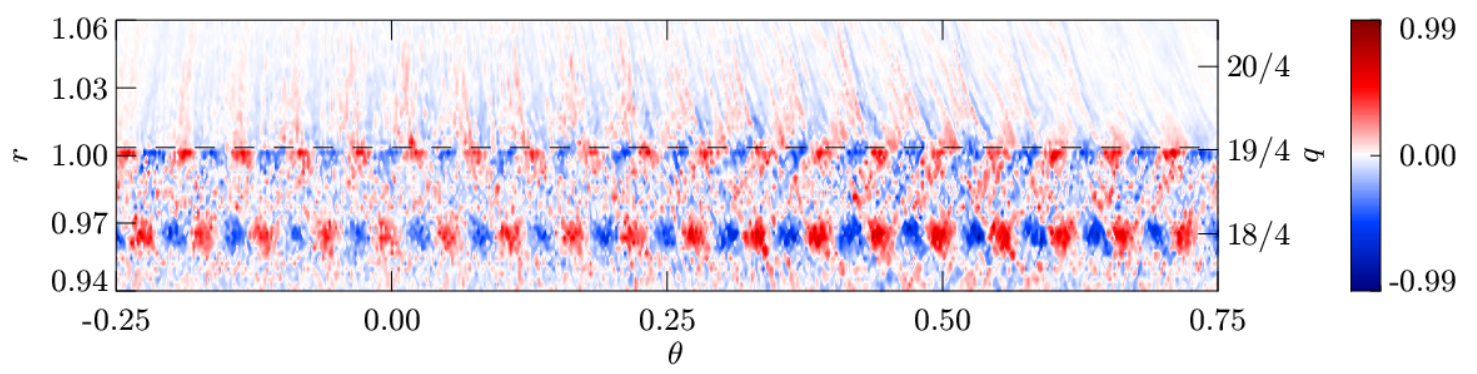

(a) $A_{0}=100$

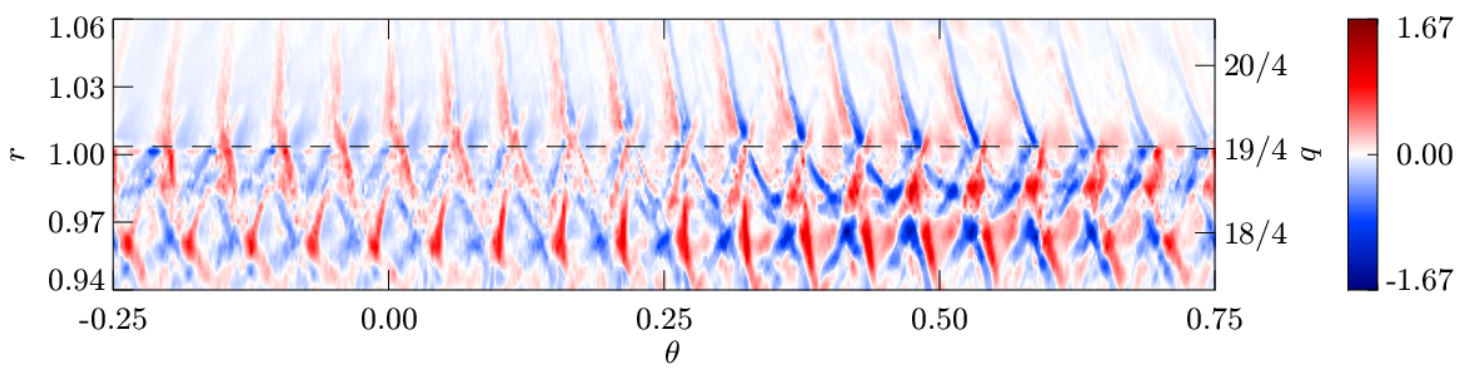

(b) $A_{0}=200$

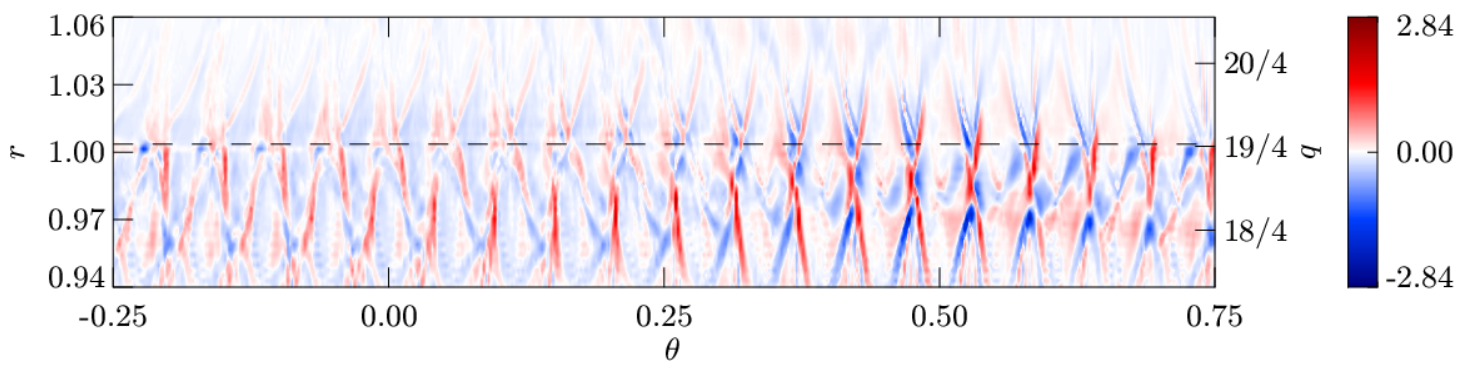

(c) $A_{0}=400$

FIG. 2: RMP-induced variation of the stationary part of the parallel current fluctuations in the poloidal plane. The fluctuations were computed by subtracting the toroidal mean as $\tilde{J}_{\|}=J_{\|}-\left\langle J_{\|}\right\rangle_{y_{k}}$. The dashed lines mark the $q=19 / 4$ position.

\section{B. Thermal state variables}

In the following, we discuss the effects of RMPs on thermal state variables $\left(n_{\mathrm{e}}, n_{\mathrm{i}}, T_{\mathrm{e}}\right.$, $\left.T_{\mathrm{e} \perp}, T_{\mathrm{i} \|}, T_{\mathrm{i} \perp}\right)$. Fig. 4 shows time-averaged zonal profiles of densities and temperatures. For each of the thermal state variables, RMPs give rise to a flattening of the profiles in the CFS-region. In the SOL, the density profiles are slightly increased by RMPs, while the temperature profiles remain nearly unchanged. Note that the changes in the profiles increase with increasing RMP-amplitude. Furthermore, the changes are not confined to resonant flux 


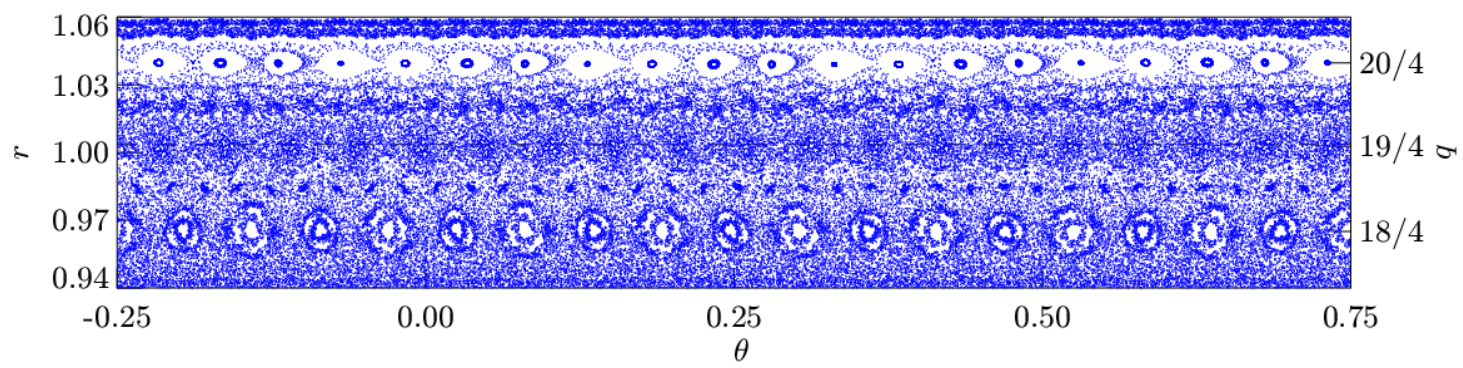

(a) $A_{0}=100$

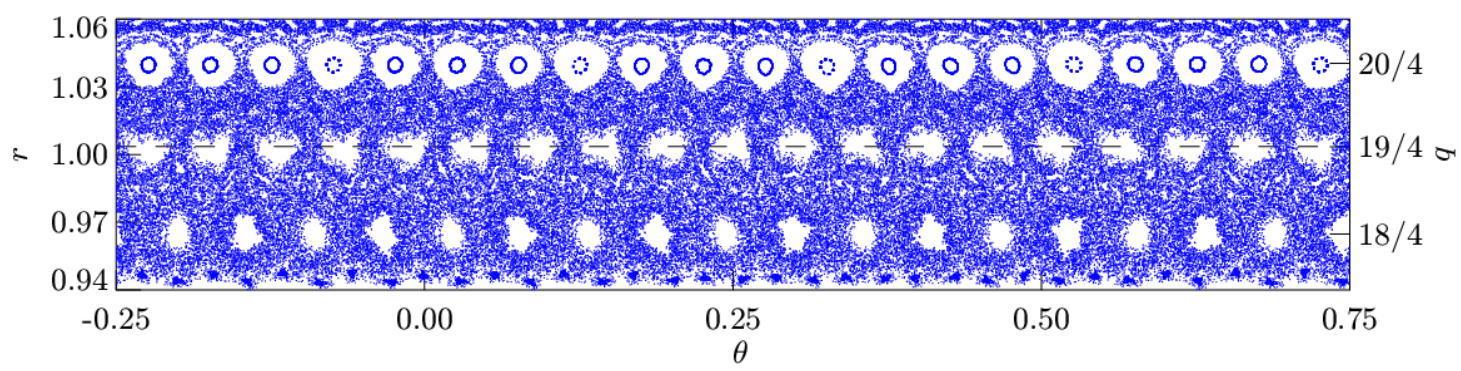

(b) $A_{0}=200$

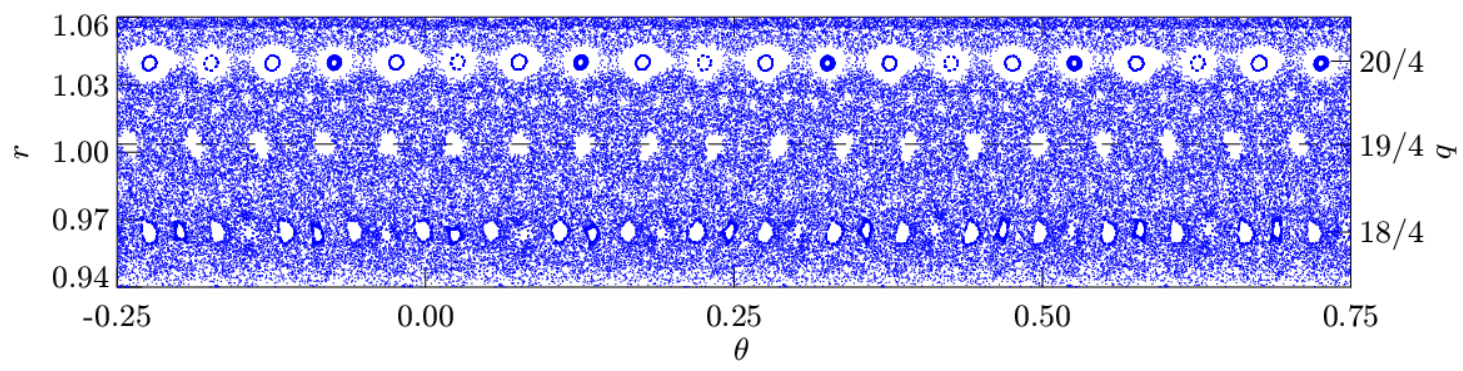

(c) $A_{0}=400$

FIG. 3: Poloidal Poincaré sections of the stationary part of the magnetic flutter field for various RMP amplitudes. 40 magnetic field lines were followed over 4000 toroidal turns.

The dashed lines mark the $q=19 / 4$ position.

surfaces but involve the entire radial simulation domain.

Fig. 5 illustrates RMP-induced changes in toroidal mode number spectra of the electron density. The total density as well as the stationary and the temporally fluctuating parts according to Eq. (10) are shown for both CFS region and SOL. The spectra of the total fluctuations in the CFS region indicate that RMPs give rise to the formation of density structures with mode numbers which are resonant with the applied RMP fields. While these resonant structures are especially pronounced in the stationary part, they do not occur in 


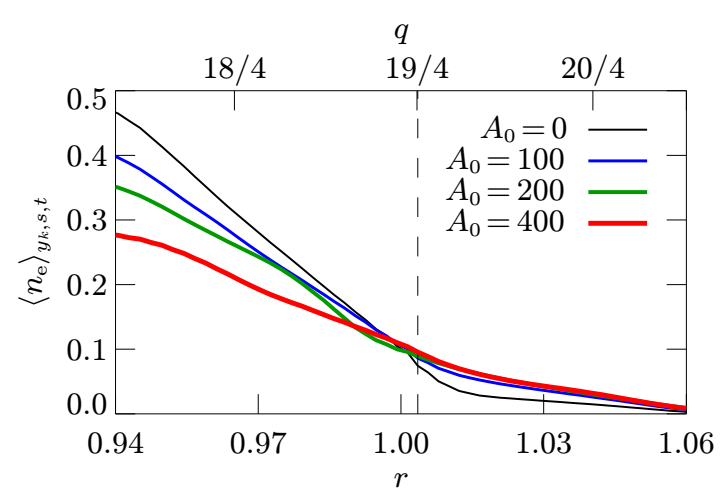

(a)

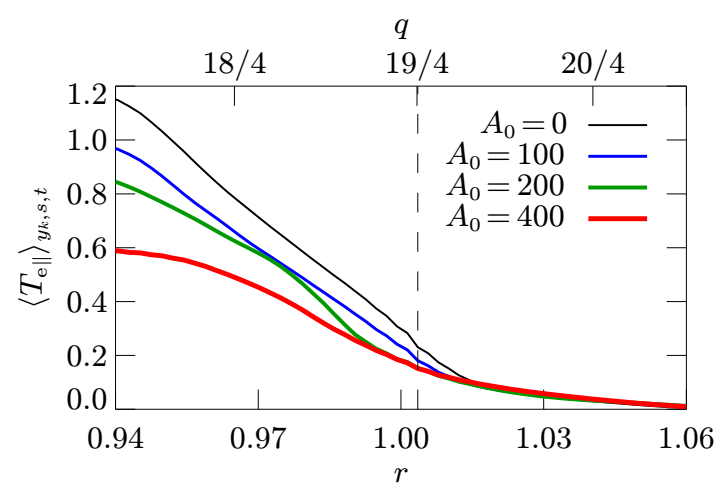

(c)

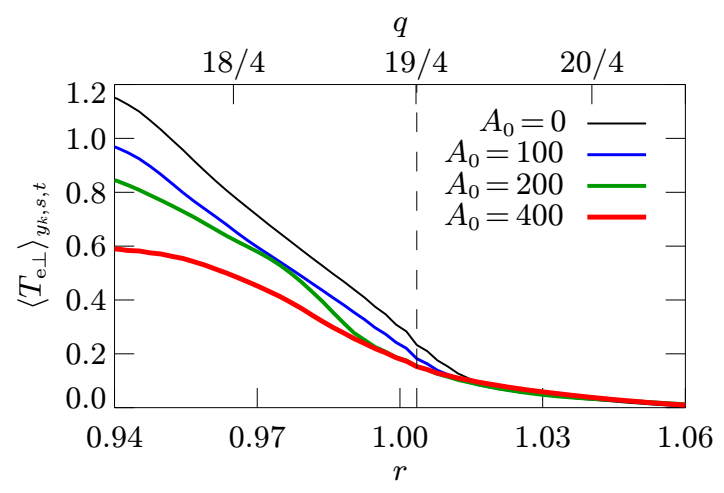

(e)

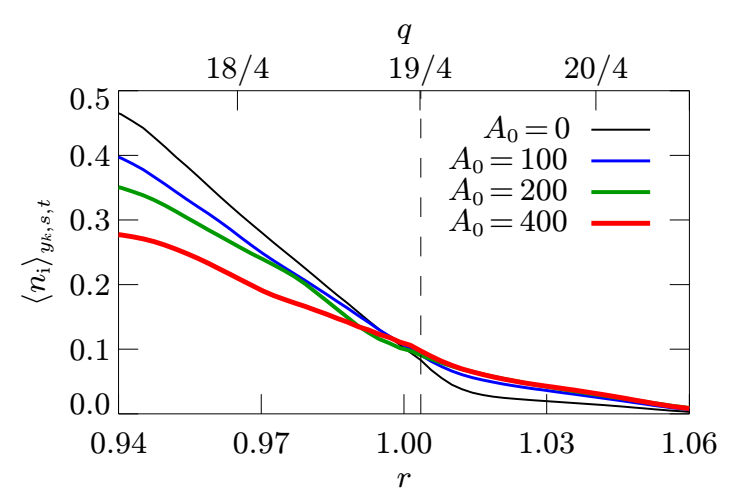

(b)

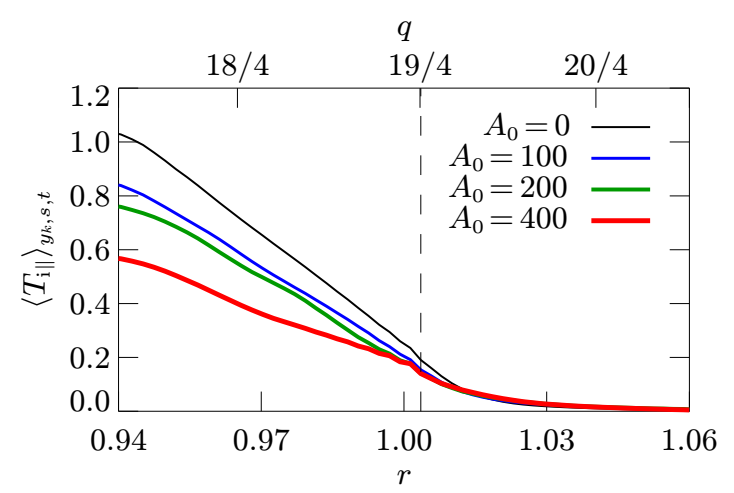

(d)

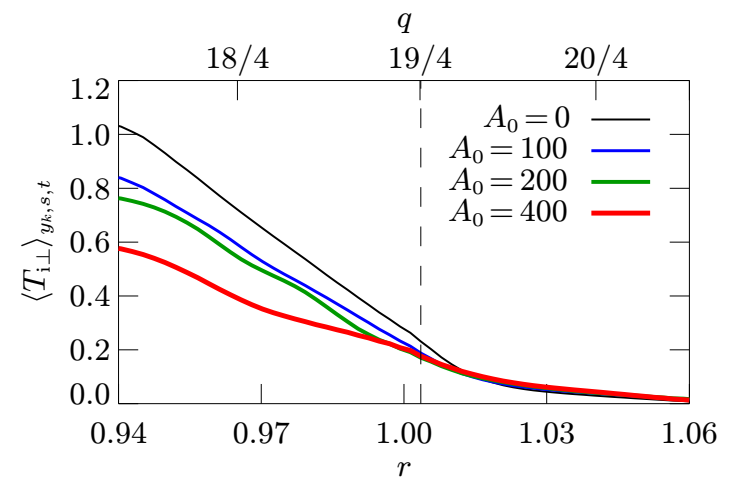

(f)

FIG. 4: Time- and flux-surface-averaged radial profiles of (a) electron density, (b) ion density, (c) parallel electron temperature, (d) parallel ion temperature, (e) perpendicular electron temperature and (f) perpendicular ion temperature for various RMP amplitudes.

The dashed lines mark the $q=19 / 4$ position. 
the temporally fluctuating part.

Thus, the imposed resonant structures are static and this is reflected in the resulting stationary component, but not in the fluctuations [17]. The temporally fluctuating part of the density in the CFS region decreases with increasing perturbation amplitude. Accordingly, the RMP fields amplify the resonant modes but attenuate other modes. The SOL exhibits

the same stationary resonant structures, although at lower amplitudes. In contrast to the CFS region, the temporally fluctuating parts of the density are not significantly attenuated in the SOL. The effects of RMPs on other thermal state variables are found to be very similar.

The results on the static component are in agreement with earlier work by Reiser et al. on RMP effects on local isothermal drift-Alfvén edge turbulence, who first found that "strong resonant effects can be attributed to (quasi-) static contributions of the perturbations" [17].

The fluctuations in the thermal state variables reflect the three-dimensional magnetic equilibrium imposed by RMPs. The RMP-induced toroidal variation of the magnetic equilibrium modifies the diamagnetic equilibrium current $\boldsymbol{J}_{*} \propto \boldsymbol{B} \times \boldsymbol{\nabla}$. This impacts the electric potential and consequently the thermal state variables [17].

\section{Convective and magnetic transport}

In order to address the question of how RMPs influence the radial transport, we compare the RMP-induced effects on the convective and magnetic transport of electron heat, in differential form given by

$$
\begin{aligned}
\mathrm{d} Q_{\mathrm{E}, \mathrm{e}} & =\frac{3}{2} p_{\mathrm{e}} u_{\mathrm{E}}^{x} \mathrm{~d} y_{k} \mathrm{~d} s \\
\mathrm{~d} Q_{\mathrm{M}, \mathrm{e}} & =q_{\mathrm{e} \|} b^{x} \mathrm{~d} y_{k} \mathrm{~d} s
\end{aligned}
$$

where $u_{E}^{x}=B^{-2} \boldsymbol{B} \times \boldsymbol{\nabla} \phi \cdot \nabla x$ denotes the radial component of the $E \times B$ drift velocity and $b^{x}=-B^{-2} \boldsymbol{B} \times \boldsymbol{\nabla} A_{\|} \cdot \boldsymbol{\nabla} x$. The radial transport of ion heat and density fluctuations are found to exhibit similar characteristics.

Fig. 6 shows time- and flux-surface-averaged radial profiles of the convective and magnetic radial transport of electron heat for various RMP amplitudes. A comparison of the magnitudes shows that the magnetic transport is negligibly small for all perturbation amplitudes. Even for ergodic vacuum perturbation fields, the radial transport is dominated 


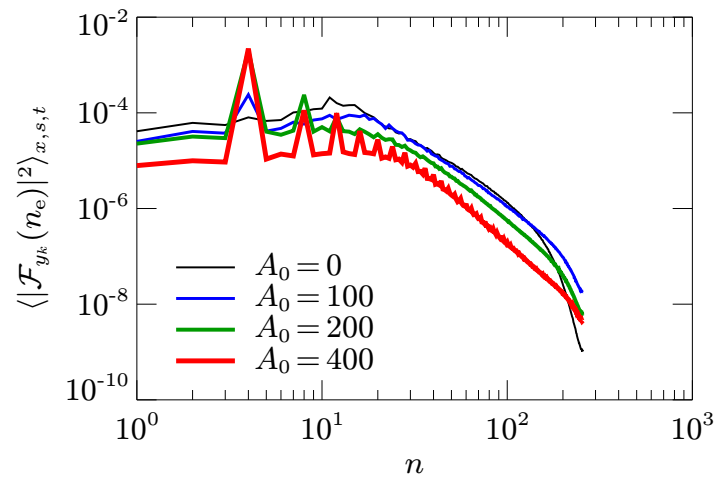

(a) Total density, CFS

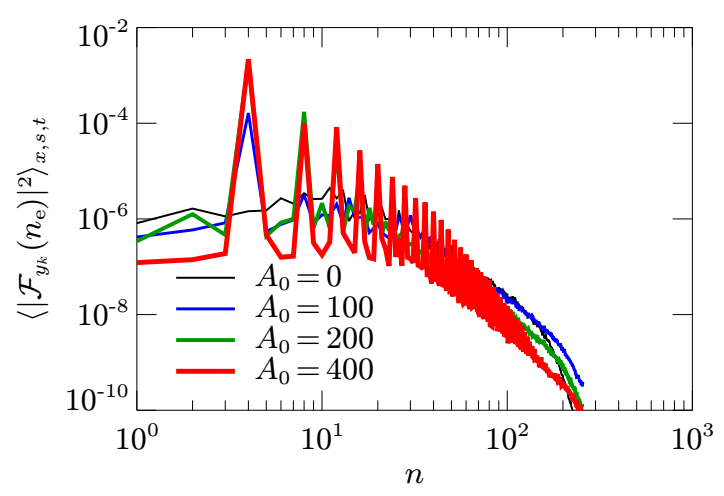

(c) Stationary part, CFS

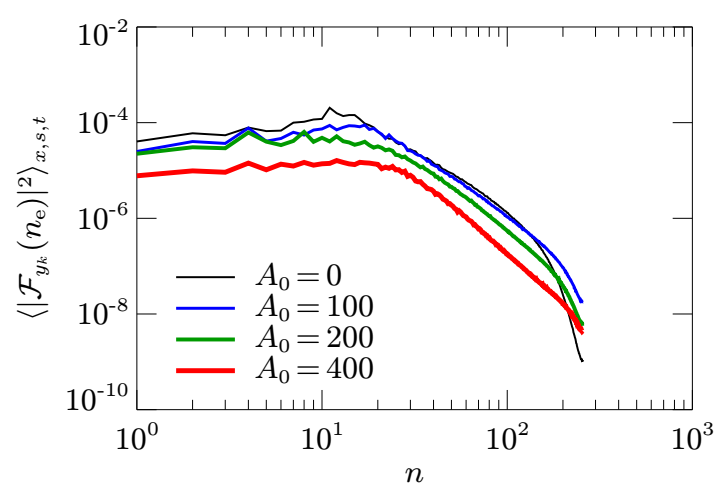

(e) Temporally fluctuating part, CFS

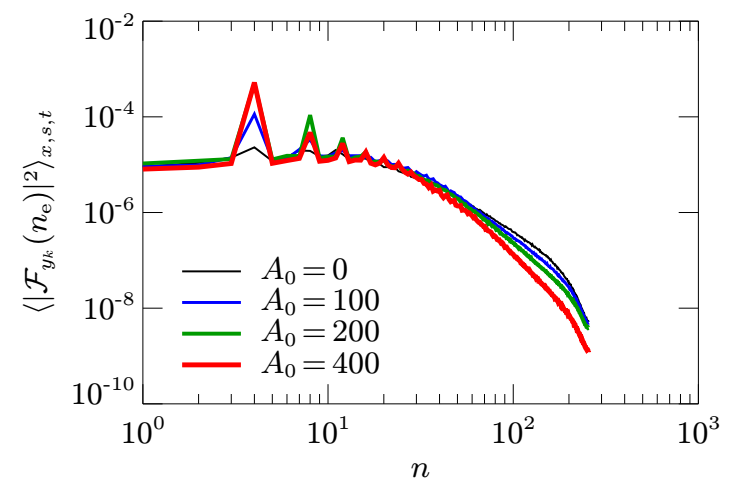

(b) Total density, SOL

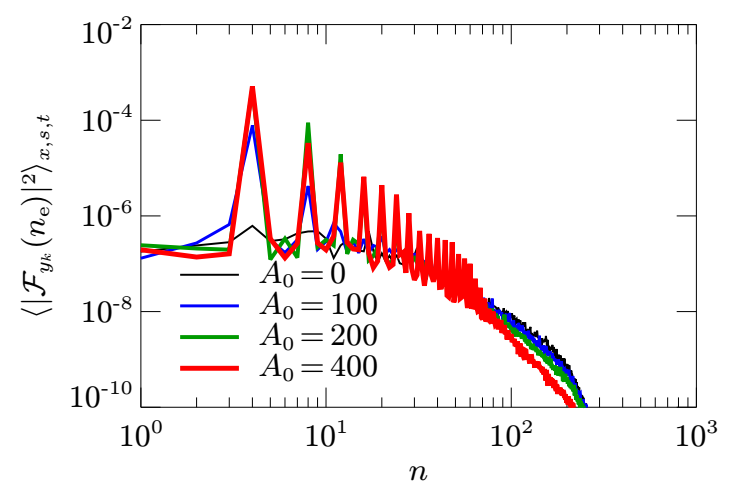

(d) Stationary part, SOL

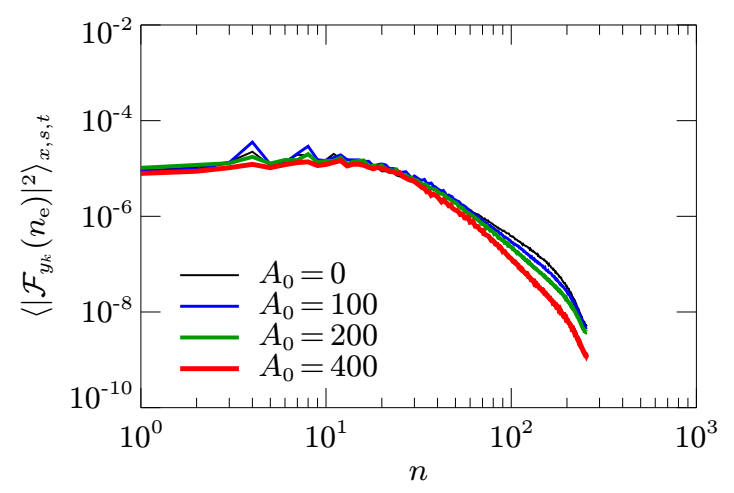

(f) Temporally fluctuating part, SOL

FIG. 5: Time- and space-averaged toroidal mode number spectra of the electron density for various RMP amplitudes. The (a, b) total density and the $(c, d)$ stationary and $(e, f)$ temporally fluctuating parts in both CFS region an SOL are shown. 


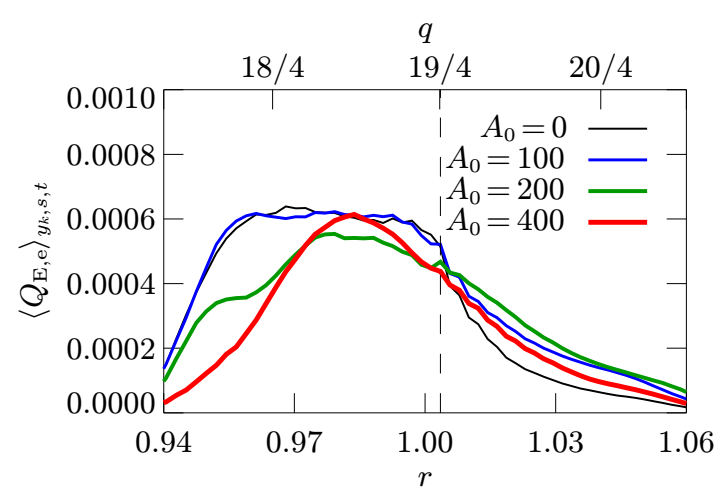

(a)

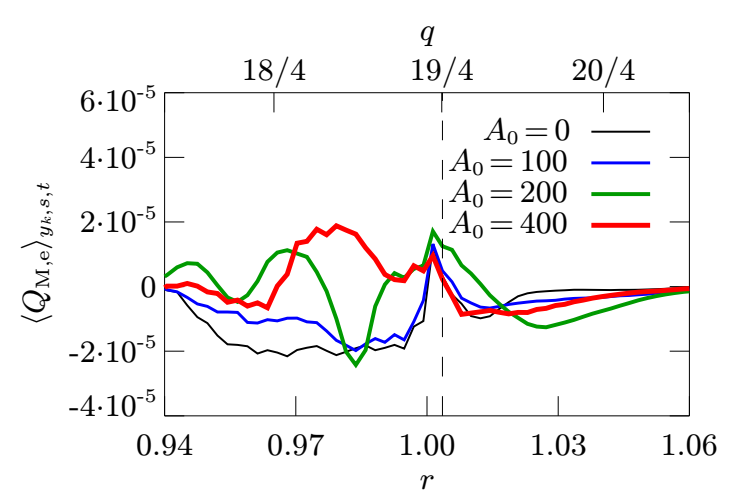

(b)

FIG. 6: Time- and flux-surface-averaged radial profiles of the (a) convective and (b) magnetic radial transport of electron heat for various RMP amplitudes.

by turbulent convection. Note that this is in agreement with the observed screening of the vacuum perturbation fields [19].

Considering Fig. 6] we can conclude that the sum of convective and magnetic transport varies somewhat with the applied perturbation amplitude. As all simulations include the same source flux and the physical dissipation terms are found to be nearly unchanged across different simulations, this effect can be ascribed to the varying transport losses through the outer edge boundary layer.

In the following we consider the modification of the convective transport by RMPs. Fig. 7 illustrates the toroidal structure of the time- and space-averaged convective transport of electron heat. Toroidal mode number spectra of the total transport $\left(p_{\mathrm{e}} \boldsymbol{u}_{\mathrm{E}}\right)$ as well as the contributions resulting from the stationary $\left(\left\langle p_{\mathrm{e}}\right\rangle_{t}\left\langle\boldsymbol{u}_{\mathrm{E}}\right\rangle_{t}\right)$ and temporally fluctuating $\left(\tilde{p}_{\mathrm{e}} \tilde{\boldsymbol{u}}_{\mathrm{E}}\right)$ parts of the dependent variables are shown for both CFS region and SOL. In the CFS region, the toroidal transport is composed of the smooth temporally fluctuating part and the comblike structure resulting from the stationary contribution. The temporally fluctuating part decreases with increasing perturbation amplitude. This decrease is partly compensated by the stationary part. Hence, the application of RMPs gives rise to a reorganisation of the total radial transport in terms of stationary convection cells. In the SOL, the effects of RMPs are similar. However, the RMP-induced reduction of the turbulent transport is less pronounced in the SOL. 


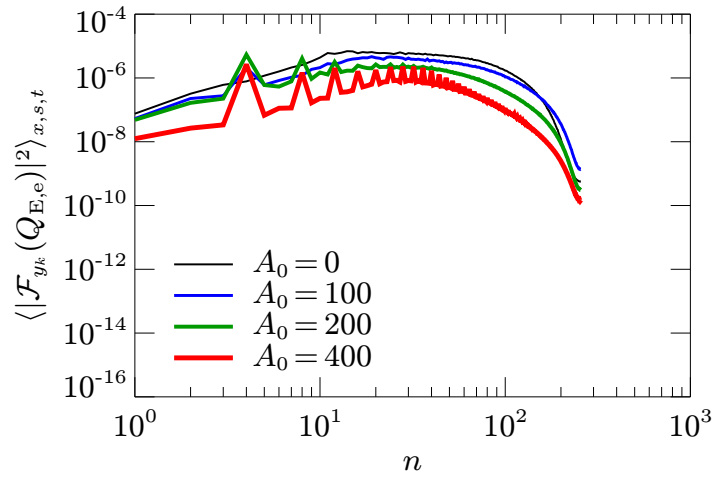

(a) Total transport, CFS

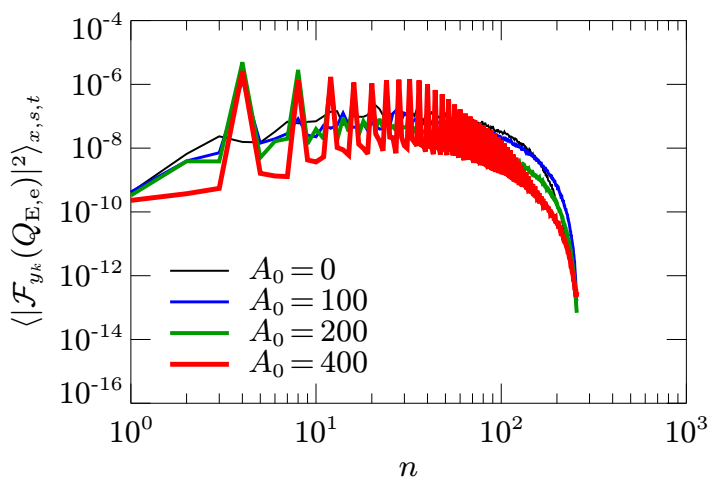

(c) Stationary part, CFS

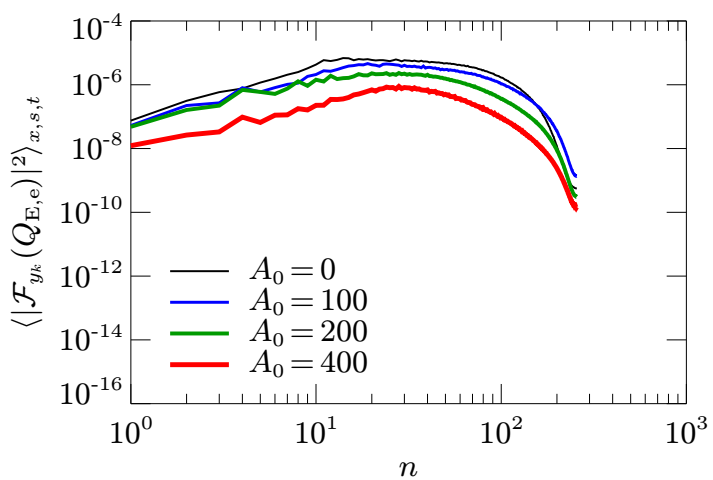

(e) Temporally fluctuating part, CFS

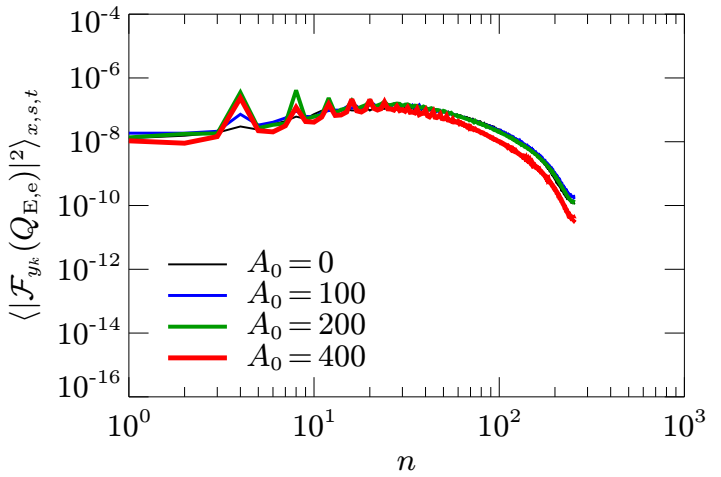

(b) Total transport, SOL

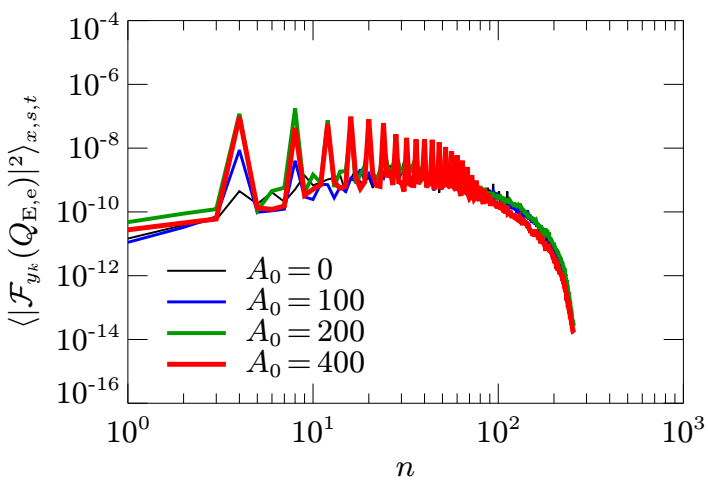

(d) Stationary part, SOL

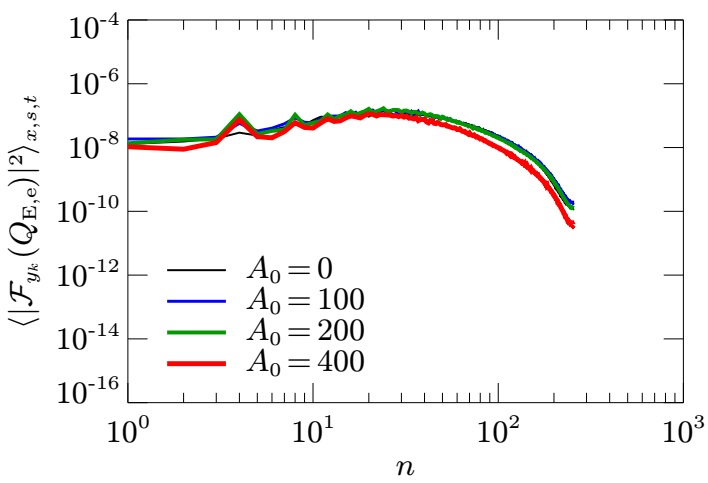

(f) Temporally fluctuating part, SOL

FIG. 7: Time- and space-averaged toroidal mode number spectra of the convective transport of electron heat for various RMP amplitudes. The (a, b) total transport and the $(c, d)$ stationary and $(e, f)$ temporally fluctuating parts in both CFS region an SOL are shown. 


\section{Poloidal plasma rotation and GAMs}

RMPs are found to influence the radial profiles of the electric potential. In the following, we quantify the associated changes in the poloidal $E \times B$ rotation velocity $\hat{u}_{\mathrm{E}}^{y_{k}}=\boldsymbol{u}_{\mathrm{E}} \cdot \hat{\mathbf{e}}_{y_{k}}$, where $\hat{\mathbf{e}}_{y_{k}}$ denotes the unit vector perpendicular to both $\mathbf{e}_{s}$ and $\mathbf{e}_{x}$. Fig. 8 shows time- and flux-surface averaged radial profiles of the squared poloidal $E \times B$ velocity. It is shown that the application of RMPs involves a reduction of the poloidal plasma rotation. Note that the average poloidal velocity in the SOL is small so that the changes mainly concern the CFS region. The presented simulation setup does not allow to draw clear conclusions about the effects of RMPs on geodesic acoustic modes (GAMs).

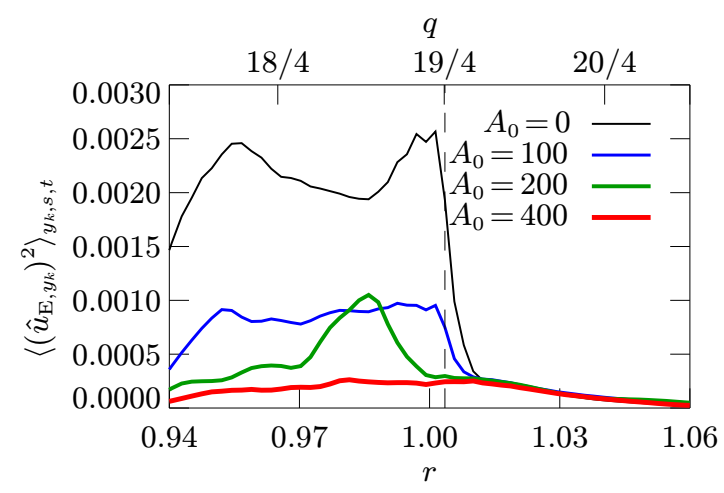

FIG. 8: Time- and flux-surface-averaged radial profiles of the squared poloidal $E \times B$ rotation velocity for various RMP amplitudes. The dashed lines mark the $q=19 / 4$ position.

\section{E. Summary and discussion of turbulence results}

The effects of non-axisymmetric RMPs were investigated by numerical simulations employing the nonlinear gyrofluid electromagnetic model GEMR. The simulation setup was arranged for typical L-mode conditions of the AUG tokamak. RMPs were implemented in terms of a magnetic perturbation potential satisfying the constraint of zero additional plasma current. The simulations were performed for three amplitudes of a multiple RMP field including the helicity components $m / n=18 / 4, m / n=19 / 4$, and $m / n=20 / 4$. The simulation results were discussed in terms of time and space averaged quantities. For the 
evaluation, the dependent variables were separated into an RMP-induced stationary component and a temporally fluctuating, turbulent contribution.

The main findings can be summarised as follows:

1. RMPs give rise to the formation of resonant, parallel plasma response currents which are radially-localised around resonant magnetic flux surfaces [19].

2. The vacuum RMP fields are effectively screened by the plasma response. The magnetic islands associated with the vacuum RMP fields are closed and replaced by islands which are poloidally shifted by half a poloidal island width.

3. The magnitude of the intrinsic magnetic flutter is reduced by up to $73 \%$ with respect to the vacuum RMP fields. Radial and poloidal profiles of the intrinsic magnetic flutter significantly differ from the imposed vacuum RMP fields.

4. RMPs cause a non-localised flattening of density and temperature profiles.

5. RMPs reduce the temporally fluctuating part of the electron density. This decrease is compensated by an RMP-induced formation of stationary, resonant density perturbations which reflect the three-dimensional magnetic equilibrium imposed by RMPs.

6. RMPs give rise to the formation of stationary convection cells which compensate the RMP-induced decrease of the transport associated with the temporally fluctuating parts of the dependent variables.

7. The magnetic transport is negligibly small compared to the convective transport. Ergodic vacuum perturbation fields do not give rise to an increased magnetic transport. This is in agreement with the screening of RMPs by the plasma response.

8. RMPs involve an attenuation of the poloidal $E \times B$ rotation.

The simulations were repeated for a multiple helicity RMP field located on the low-field side at $\theta=0$, and a single RMP exhibiting no poloidal localisation. We found that the main effects (formation of resonant plasma fluctuations, reorganisation of the turbulent transport in terms of resonant convection cells, negligible magnetic transport) do not depend on either the poloidal localisation or the number of included helicity components of the applied RMP field. 
In the present GEMR modeling, the major novel results concern the self-consistent temperature dynamics of both electrons and ions. Thus, convective and magnetic transport can be evaluated in a self-consistent model.

The screening of RMPs by plasma response currents was recently discussed in Refs. [16, 19]. In both works, the screening effect was ascribed to the formation of radially-localised current layers in phase with the RMP field. Moreover, out-of-phase currents leading to the growth of poloidally shifted magnetic islands were reported [19]. Our simulations reproduce both the complete screening of the vacuum perturbation fields by in-phase currents and the formation of poloidally shifted magnetic islands by out-of-phase currents. The RMP-induced formation of stationary density fluctuations and resonant convection cells was previously reported in Refs. [15, 17, 18] and is in good agreement with our results. Furthermore, our results confirm an RMP-induced reduction of the zonal averaged poloidal $E \times B$ velocity as reported in Ref. [15]. By contrast, our simulation setup does not lead to an RMP-induced increase of GAMs as found in [28]. Note however that the results in [28] were obtained by particular adapted equations.

The RMP-induced decrease of the zonal density and temperature gradients in our simulations could be an important indication on the mechanism governing the mitigation of ELMs by RMPs. Edge turbulence models with self-consistent profiles do not exhibit transport barriers when no further mechanisms are present. Unlike MHD interchange models, the two-fluid and gyrofluid models specifically contain finite-beta drift-wave and ion temperature effects. These nonlinear drift wave instabilities always supersede linear instabilities, hence there is no threshold character [20]. Thus, the effects of RMPs on H-mode configurations can not be studied in a self-consistent manner. Assuming that RMP-induced changes are similar in L- and H-mode plasmas, the decrease of the zonal pressure gradient could explain the mitigation and suppression of pressure-gradient-driven ELMs.

In sum, we can conclude that our simulation results generally agree with previous studies. Significant discrepancies can be ascribed to differences in the basic model assumptions (local flux tube model without SOL versus global geometry model including profile evolution and SOL). The heat transport associated with a self-consistent temperature dynamics (not included by previous models) was found to exhibit similar characteristics to the density transport. Moreover, the magnetic contribution to the radial transport was found to be negligibly small even for strongly ergodised vacuum RMP fields. 


\section{BALLOONING MODE BURST SIMULATIONS}

In the following we analyse the use of computational setups within the present gyrofluid approach to model ELMy H-mode like scenarios including RMP fields.

The computations discussed in this section are based on the ideal ballooning unstable simulation setup described in Refs. [21, 22]. The initial safety factor profile is defined as $q_{0}=1.41+3.29(r / a)^{2}$, so that the flux surface with $q=m / n=18 / 4$ coincides with the position of the steepest gradients at $r / a=0.97$.

The local parameters, taken as mid pedestal values, correspond to electron and ion temperatures $T_{e}=300 \mathrm{eV}, T_{i}=360 \mathrm{eV}$, densities $n_{e}=n_{i}=2.5 \cdot 10^{19} \mathrm{~m}^{-3}$, magnetic field strength $B=2.0 \mathrm{~T}$, major torus radius $R=1.65 \mathrm{~m}$, aspect ratio $R / a=3.3$, perpendicular temperature gradient length $L_{T}=L_{\perp}=3.0 \mathrm{~cm}$, density gradient length $L_{n}=6.0 \mathrm{~cm}$. The radial domain of the simulations covers a range of $L_{\perp}$ on either side of the LCFS. As above, $n_{y}=512$ perpendicular mesh points are used, and the radial domain with $n_{x}=64$ spans the plasma edge region between the H-mode pedestal top, with plasma core parameters as inner boundary values, and the outer bounded scrape-off layer region $(r / a=1 \pm 0.06)$. This translates to a ratio $\delta=\rho_{s} / a=0.0026$ between ion gyroradius $\rho_{s}$ and minor torus radius $a$, and a local plasma beta of $\beta_{e 0}=7.5 \cdot 10^{-4}$.

The initial profiles now correspond to a typical AUG (unmitigated) ELMy H-mode scenario. We apply the RMP field (as described at the end of sec. 2) to the initialised profiles.

The RMP amplitudes are gives the values $1.0 \cdot 10^{-7} \mathrm{Tm}\left(A_{0}=0.1\right), 1.0 \cdot 10^{-6} \mathrm{Tm}\left(A_{0}=1\right)$, $1.0 \cdot 10^{-5} \mathrm{Tm}\left(A_{0}=10\right), 2.0 \cdot 10^{-5} \mathrm{Tm}\left(A_{0}=20\right)$, and $4.0 \cdot 10^{-5} \mathrm{Tm}\left(A_{0}=40\right)$. The corresponding magnetic perturbation fields are of order $10^{-6} \mathrm{~T}\left(A_{0}=0.1\right), 10^{-5} \mathrm{~T}\left(A_{0}=1\right)$, and $10^{-4} \mathrm{~T}\left(A_{0}=10-40\right)$,

In the absence of RMP fields the initialised pedestal profiles are ideal ballooning unstable and show single ELM-like bursts as discussed in Refs. [21, 22]. In the following, effects of added RMP fields on this ideal ballooning blow-out scenario are studied.

\section{A. Effects of RMPs on ideal ballooning unstable H-mode states}

We consider the "H-mode ELM" scenario of Refs. [21, 22] and focus on the evolution of an ideal ballooning blow-out, not including any profile sustaining sources. In the RMP-free 
case, the prepared pedestal state evolves into an ideal ballooning instability with toroidal mode number $n=7$. If RMPs are applied, the plasma adjusts to the RMP-induced threedimensional magnetic equilibrium [17], and the most unstable mode number associated with the RMP-free nominal case $(n=7)$ is found to be replaced by a mode number which is resonant with the perturbation fields. Below, we refer to this ideal ballooning scenarios as an ELM model, while recognising its provisional status.

Fig. 9 illustrates the time evolution of space-averaged toroidal mode number spectra associated with the ELM-induced convective radial transport of electron heat as defined by Eq. (11). In order to make the linear phase visible, the spectra are time-dependently normalized to their maximum values. In the RMP-free case (9a), the most unstable mode is the one with $n=7$. For the lowest perturbation amplitude $A_{0}=0.1$ (9b), the initial linear phase for $t \lesssim 15$, is superseded by the RMP-induced resonant mode number $n=4$. Later, for $t \gtrsim 15$, the nominal mode with $n=7$ competes with a resonant mode with $n=8$. For $A_{0}=1$ (9c), the nominal mode is completely replaced by RMP-induced resonants, and for the largest RM perturbation amplitude $A_{0}=10(9 \mathrm{~d})$, resonant modes even prevail the nonlinear dynamics.

Fig. $9 \mathrm{~b}$ shows a situation where the nominal mode with $n=7$ competes with the RMPinduced resonant mode with $n=8$. The interaction between two competing neighbouring modes could have a mitigative effect on the ideal ballooning ELM blow-out. In Fig. 10 , we investigate this point by considering time traces of the volume-averaged convective and magnetic radial transport of electron heat as given by Eqs. 11112 . The maximum of the convective heat transport (Fig. 10a) is shifted from $t \approx 22$ in the perturbation-free case to $t \approx 10$ for the highest perturbation amplitude $A_{0}=10$. Moreover, the amplitude of the peak transport is slightly increased with increasing perturbation amplitude. The magnetic transport (Fig. 10b) exhibits similar characteristics: RMPs cause a shift of the peak transport to earlier times and give rise to a significant increase of the magnetic transport in the linear phase. For the perturbation-free case, the magnetic contribution to the transport in the linear phase is negligible. By contrast, magnetic and convective transport become comparable if a magnetic perturbation is applied. As the RMP-free linear ballooning instability substantially preserves the magnetic flux surfaces (the associated magnetic flutter emerges only in the nonlinear phase), this effect can be ascribed to the RMP-induced formation of ergodic field regions which enhance the magnetic transport in the linear phase. 


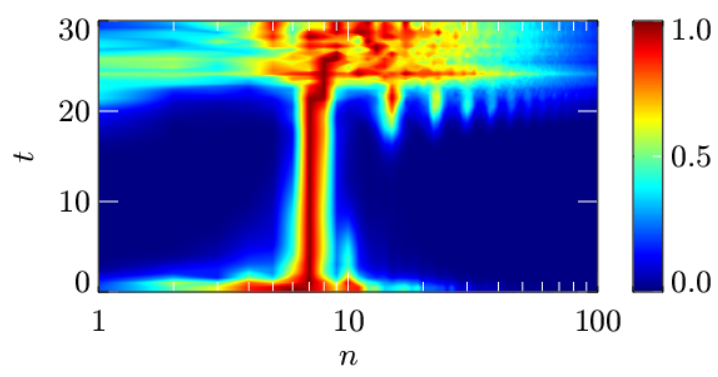

(a) $A_{0}=0$

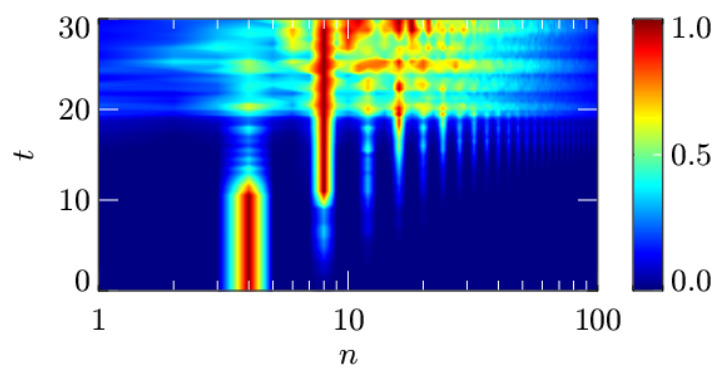

(c) $A_{0}=1$

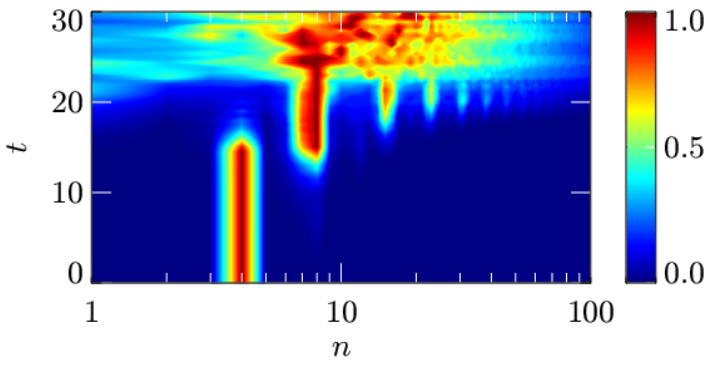

(b) $A_{0}=0.1$

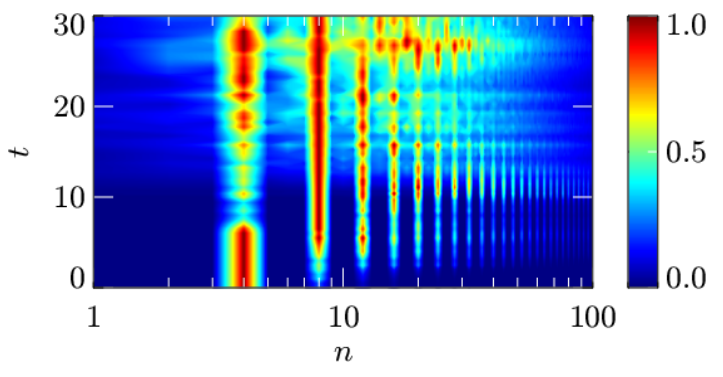

(d) $A_{0}=10$

FIG. 9: Space-averaged toroidal mode number spectra associated with the time evolution of the convective radial transport of electron heat for various RMP amplitudes. For better visualisation, the transport is normalized to its maximum value at each time slice.

A mitigation of the ideal ballooning ELM blow-out due to a competition between the most unstable mode associated with the RMP-free case and the RMP-induced resonant modes is not observed. On the contrary, RMPs involve the formation of stationary density fluctuations [17], which drive the growth of a resonant MHD component. This can be explained by the fact that the amplitudes of the quasi-turbulent fluctuations associated with the initially prescribed pedestal state are small so that the RMP-induced resonant structures can easily prevail. If the initial, quasi-turbulent density fluctuations are increased by a factor of 1000 , the linear growth phase of the nominal mode with $n=7$ is shortened so that this mode is dominant up to perturbation amplitudes $A_{0} \leq 1$. For $A_{0}=10$, we again observe a competition between the modes with $n=7$ and $n=8$. However, a mitigation of the ideal ballooning ELM blow-out is not observed.

The above results indicate that the present ideal ballooning unstable pedestal profile scenario is not appropriate to investigate a possible RMP-induced mitigation effect on ELMs. 


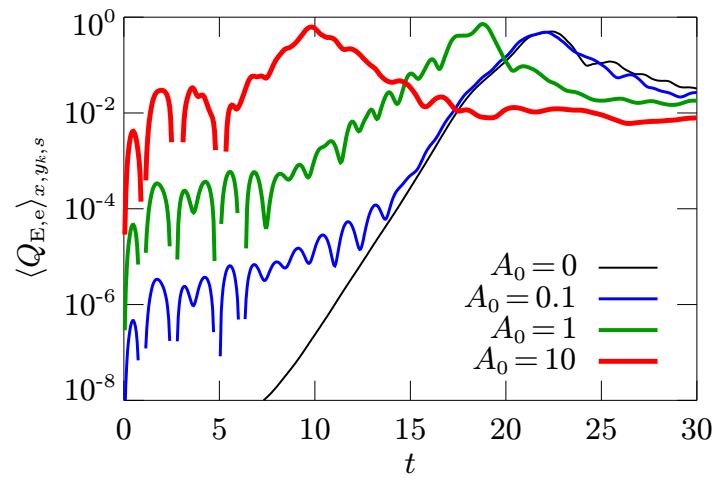

(a)

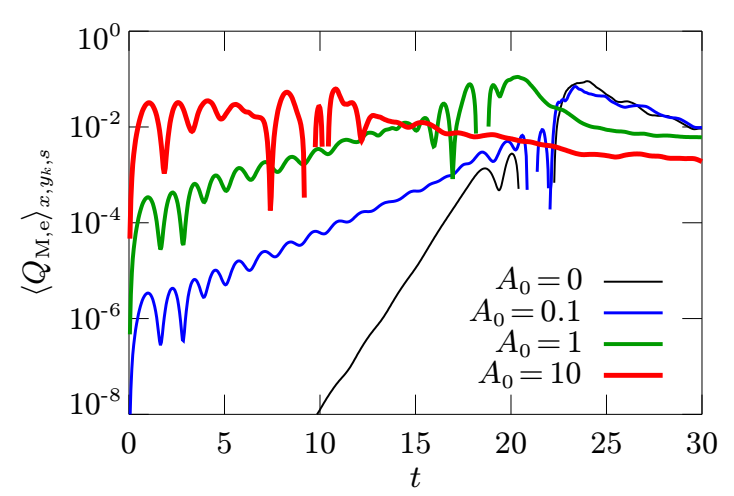

(b)

FIG. 10: Time variation of the volume-averaged (a) convective and (b) magnetic radial transport of electron heat for various RMP amplitudes.

\section{B. Summary and discussion of results on RMP suppression of edge localized bursts}

The effects of RMPs on ideal ballooning unstable edge profiles were examined. An "Hmode" model scenario described in [21, 22] was considered.

The main findings can be summarized as follows:

1. Within the ideal ballooning unstable pedestal scenario, the RMP-induced formation of resonant structures drives the formation of ballooning blow-outs which are resonant with the magnetic perturbation field.

2. RMPs cause a modification of the zonal profiles of electron density and electric potential by up to $30 \%$. The temporal fluctuations of the zonal profiles are strongly reduced.

3. The examined "H-mode"-like model scenarios do not allow to simulate a mitigation of ideal ballooning ELMs by RMPs.

The above simulations constitute a first approach to study the effects of RMPs on ELMlike bursts within the gyrofluid electromagnetic model GEMR. A direct mitigation of edge loclaised ideal ballooning modes could not be established in the simulations. On the contrary, RMPs were found to drive the formation of ideal ballooning ELMs. In Ref. [29] a 
destabilization of ELMs by RMPs in otherwise quiescent H-mode states has actually been reported. However, none of the proposed destabilization mechanisms is in agreement with the RMP-induced formation of resonant structures which drive resonant IBM blow-outs.

\section{CONCLUSIONS}

We have considered the effects of externally-applied resonant magnetic perturbations (RMPs) on tokakamak edge turbulence and ideal ballooning bursts in 6-moment electromagnetic gyrofluid computations including zonal profile evolution.

The interpretation of the L-mode like turbulence simulation results leads to the following physical picture. RMPs give rise to plasma response currents which screen the vacuum perturbation fields. Even for strongly ergodized vacuum perturbation fields, the amplitude of the intrinsic magnetic flutter and the resulting intrinsic ergodicity are hardly changed by RMPs. As a consequence, the radial transport by parallel motion along radially perturbed magnetic field lines is not increased. Even for strongly ergodic vacuum perturbation fields, the radial transport is mainly due to fluid-like $E \times B$ convection. As the plasma adjusts to the RMP-induced three-dimensional magnetic equilibrium, the convective transport is reorganized in terms of convection cells which are resonant with the toroidal and poloidal mode numbers of the perturbation fields. Accordingly, the radial convective transport exhibits a stationary component which increases with increasing RMP amplitude. For the same reason, RMPs give rise to a decrease of the turbulent fluctuations in the thermal state variables, whereas the stationary contributions to the fluctuations increase. Moreover, the RMP-induced stationary structures decelerate the poloidal plasma rotation. Except for the imposed stationary structures, the drift-wave mode structure of the turbulent fluctuations is widely preserved.

The computations faced the problem that the artificial dissipation slightly varied with the RMP amplitude. Consequently, the total (convective and magnetic) transport was not completely preserved across the simulations. The problem was increased by an additional boundary dissipation which was found to be necessary for stable RMP simulations. The main findings (screening of RMPs by plasma response currents, dominance of the convective over the magnetic transport) are not affected by this constraint. By contrast, a comparison between the absolute values of the fluctuations in the dependent variables has to be inter- 
preted carefully. Nevertheless, the tendency for an RMP-induced decrease of the fluctuations is clear.

If RMPs are applied to an ideal ballooning unstable "H-mode"-like initial pedestal configuration, the profiles adjusts to the RMP-induced three-dimensional magnetic equilibrium and form resonant perturbations in the dependent variables. The interchange-ballooning drive increases the resonant perturbations so that they can grow faster than the most unstable mode of the perturbation-free case.

The presented simulations constitute a first gyrofluid approach towards a simulation of RMP effects on edge turbulence and on ELM mitigation. A direct mitigation of ideal ballooning mode bursts in modelled "H-mode"-like states could not be observed.

In turbulence computations the RMP fields were found to considerably reduce the pedestal profile gradients (to around a half for typical experimental perturbation field strengths). Following this picture, ELMs are likely suppressed if in an experimental Hmode scenario the edge profiles are sustained below a ballooning unstable critical gradient by RMP effects on the (inter-ELM) turbulent transport.

\section{Acknowledgements}

This work was mainly supported by the Austrian Science Fund (FWF) Y398. This work has been carried out within the framework of the EUROfusion Consortium and has received funding from the Euratom research and training programme 2014-2018 under grant agreement No 633053. The views and opinions expressed herein do not necessarily reflect those of the European Commission.

\section{References}

[1] Federici G, Loarte A and Strohmayer G 2003 Plasma Phys. Control. Fusion 451523

[2] Federici G 2006 Phys. Scr. T124 1

[3] Loarte A et al. 2003 Plasma Phys. Control. Fusion 451549

[4] Evans T E et al. 2004 Phys. Rev. Lett. 92235003 
[5] Evans T E et al. 2005 Nucl. Fusion 45595

[6] Evans T E et al. 2006 Nature Phys. 2419

[7] Liang Y et al. 2007 Phys. Rev. Lett. 98265004

[8] Liang Y et al. 2010 Nucl. Fusion 50025013

[9] Kirk A et al. 2011 Plasma Phys. Control. Fusion 53065011

[10] Suttrop W et al. 2011 Phys. Rev. Lett. 106225004

[11] Suttrop W et al. 2011 Plasma Phys. Control. Fusion 53124014

[12] Tokar M Z et al. 2007 Phys. Rev. Lett. 98095001

[13] Tokar M Z et al. 2008 Nucl. Fusion 48024006

[14] Snyder P B et al. 2007 Nucl. Fusion 97961

[15] Beyer P et al. 2002 Plasma Phys. Control. Fusion 442167

[16] Bécoulet M 2012 et al. Nucl. Fusion 52054003

[17] Reiser D and Scott B 2005 Phys. Plasmas 12122308

[18] Reiser D 2007 Phys. Plasmas 14082314

[19] Reiser D and Chandra D 2009 Phys. Plasmas 16042317

[20] Scott B 2005 Phys. Plasmas 12062314

[21] Kendl A, Scott B D and Ribeiro T T 2010 Phys. Plasmas 17072302

[22] Peer J, Kendl A, Scott B D 2013 Plasma Phys. Control. Fusion 55015002

[23] Scott B D 2005 Phys. Plasmas 12102307

[24] Ribeiro T T and Scott B 2005 Plasma Phys. Control. Fusion 471657

[25] Ribeiro T T and Scott B 2008 Plasma Phys. Control. Fusion 50055007

[26] Scott B D 2006 Contrib. Plasma Phys. 46714

[27] Scott B 2001 Phys. Plasmas 8447

[28] Reiser D 35th EPS Conf. Proc. 32D P2.031

[29] Canik et al. 2004 Nucl. Fusion 50034012 